\title{
A smooth embedding domain method based on the penalty approach
}

\author{
J. Haslinger ${ }^{1}$, T. Kozubek ${ }^{2}$, and G. Peichl ${ }^{3}$ \\ ${ }^{1}$ Charles University, Prague, Czech Republic, hasling@karlin.mff.cuni.cz \\ ${ }^{2}$ VSB-Technical University of Ostrava, Czech Republic, tomas.kozubek@vsb.cz \\ ${ }^{3}$ University of Graz, Austria, gunther.peichl@uni-graz.at
}

\begin{abstract}
It is well known that convergence of the fictitious domain formulation with boundary Lagrange multipliers is slow due to the lower global regularity of its solution. The paper presents a smoothed variant of this approach which is based on a formulation in the form of a state constraint optimal control problem. The convergence rate is increased as seen from a model example.
\end{abstract}

\section{Introduction}

Fictitious domain methods represent an efficient tool for the numerical realization of elliptic equations. The idea of such methods is simple: a boundary value problem defined in $\omega$ is replaced by a new one formulated in a domain $\Omega$ having a simple shape ( a box, e.g.) and which contains $\omega$ in its interior. The new problem in $\Omega$ is defined in such a way that its solution when restricted to $\omega$ coincides with the solution of the original problem. One benefit of this approach is readily seen: we can use special (for ex. uniform) finite element partitions of $\Omega$ generating stiffness matrices with a particular structure which makes it possible to solve the resulting systems of linear algebraic equations by fast methods. A possible way to formulate the new problem in $\Omega$ is based on the use of boundary Lagrange multipliers. To illustrate the idea, we suppose that Dirichlet boundary conditions are prescribed on $\partial \omega$. This condition can be viewed as the constraint which will be treated by Lagrange multipliers defined on $\partial \omega$. Thus the new formulation in $\Omega$ involves two unknowns: the primal variable $u \in V$ and the corresponding Lagrange multiplier $\lambda \in \Lambda$ releasing the prescribed Dirichlet data on $\partial \omega$. For a discretization one can 
use standard mixed finite element methods [2]. Let $V_{h}, \Lambda_{H}$ be finite dimensional approximations of $V$ and $\Lambda$, respectively. If partitions of $\Omega$ used for the construction of $V_{h}$ do not respect the geometry of $\omega$, yet another important fact is remarkable: the stiffness matrix $A$ corresponding to $V_{h}$ does not depend on $\omega$. Thus it can be assembled and stored once for all. The only information on $\omega$ is encoded in the off-diagonal blocks of the resulting saddle-point matrix coupling the primal with dual variables. This property of $A$ can be utilized in situations when the domain $\omega$ changes many times during computations (in shape optimization, free boundary problems, etc., for details see [7, 4]). It turned out however that this fictitious domain method converges slowly due to lack of global regularity of the solution $u$ in $\Omega([3])$. The singularity of $u$ is concentrated on $\partial \omega$. To improve the convergence rate an alternative approach was proposed in [6] which is based on approximate controllability arguments. A space of controls defined on a closed curve $\Gamma$ lying in $\Omega \backslash \bar{\omega}$ was introduced which force the boundary condition on $\partial \omega$ to be satisfied with an arbitrarily small error measured in the $L^{2}(\partial \omega)$ - norm. The solution to this new formulation still has a singularity in $\Omega$ but now located on $\Gamma$ instead of $\partial \omega$. Thus in a vicinity of $\partial \omega$ the solution is smooth. This simple idea improved considerably the convergence rate. Unfortunately this new formulation and its discretization may have more than one solution, in general. Theoretically, uniqueness of the solution to the discretized problem is guaranteed provided that the distance between $\Gamma$ and $\partial \omega$ is small enough. This paper presents a new formulation which overcomes this drawback since it has a unique solution to both, the continuous as well as the discrete setting. This formulation is given by a state constrained optimization problem in which the $L^{2}(\partial \omega)$-norm of the distance between the trace of the solution and the prescribed Dirichlet data bounded from above by a given positive constant $\varepsilon$ represents the state constraint and the norm of the control variable serves as the cost functional. Taking a sequence of such optimization problems and letting $\varepsilon$ tend to zero (i.e. satisfying the boundary condition on $\partial \omega$ with increasing accuracy) and simultaneously approaching $\Gamma$ to $\partial \omega$ with an appropriate "velocity" depending on $\varepsilon$, we obtain in the limit the original (non-smooth) fictitious domain formulation with boundary Lagrange multipliers on $\partial \omega$.

The paper is organized as follows: Section 2 presents two variants of the fictitious domain formulation of non-homogeneous Dirichlet boundary value problems: the classical (non-smooth) one with Lagrange multipliers on the boundary and its smooth counterpart formulated as a state constraint optimal control problem. Section 3 contains main theoretical results. First, the stability with respect to variations of $\omega$ of the non-smooth variant of the fictitious domain approach is recalled. On the basis of this result we prove that under appropriate assumptions, the solutions to this new formulation are bounded. This enables us to pass to the limit in the 
sequence of the smooth variants and to show that the respective limit is the solution to the original non-smooth setting. To realize the state constraint problem we use a penalty technique described in Section 4. Finally, in Section 5 we present numerical results of a model example which illustrate the efficiency of this new approach. Throughout the paper we use standard notation: $H^{k}(D), k \geq 0$ integer, stands for the Sobolev space of functions which together with their generalized derivatives up to order $k$ are square integrable in $D$, i.e. they belong to $L^{2}(D)\left(H^{0}(D)\right)$. The norm in $H^{k}(D)$ is denoted by $\|\cdot\|_{k, D}$ and the scalar product in $L^{2}(D)$ by $(\cdot, \cdot)_{0, D}$.

\section{Formulation of a smooth embedding domain method}

We consider a nonhomogeneous Dirichlet boundary value problem in a bounded domain $\omega_{\gamma} \subset \mathbb{R}^{2}$ with Lipschitz boundary $\gamma:=\partial \omega_{\gamma}$ :

$$
\left.\begin{array}{rll}
-\triangle u=f & \text { in } & \omega_{\gamma}, \\
u=g & \text { on } & \gamma
\end{array}\right\}
$$

where $f \in L_{l o c}^{2}\left(\mathbb{R}^{2}\right)$ and $g \in H^{1 / 2}(\gamma)$ are given. Embedding $\omega_{\gamma}$ into a larger computational domain $\Omega$ such that $\bar{\omega}_{\gamma} \subset \Omega$, for example a rectangle (see Figure 1), the standard embedding domain approach replaces (1) by the saddle-point problem

$$
\left.\begin{array}{l}
\text { Find }(\hat{u}, \hat{\lambda}) \in H_{0}^{1}(\Omega) \times H^{-1 / 2}(\gamma) \text { such that } \\
(\nabla \hat{u}, \nabla v)_{0, \Omega}-\langle\hat{\lambda}, v\rangle_{\gamma}=(f, v)_{0, \Omega}, \quad v \in H_{0}^{1}(\Omega), \\
\langle\mu, \hat{u}\rangle_{\gamma}=\langle\mu, g\rangle_{\gamma}, \quad \mu \in H^{-1 / 2}(\gamma),
\end{array}\right\}
$$

where $H^{-1 / 2}(\gamma)$ is the dual space of $H^{1 / 2}(\gamma):=H^{1}(\Omega)_{\gamma}$ and $\langle,\rangle_{\gamma}$ stands for the duality pairing between $H^{-1 / 2}(\gamma)$ and $H^{1 / 2}(\gamma)$. The following result is well-known $([3])$.

Proposition 1. Problem (2) has a unique solution $(\hat{u}, \hat{\lambda})$. Moreover, $\hat{u}_{\omega_{\omega_{\gamma}}}$ is the solution of (1) and $\hat{\lambda}=\left[\frac{\partial \hat{u}}{\partial \nu}\right]_{\gamma}$ corresponds to the jump of the normal derivative of $\hat{u}$ across $\gamma$.

Although the extended solution $\hat{u}$ is smooth on $\omega_{\gamma} \cup\left(\Omega \backslash \bar{\omega}_{\gamma}\right)$ it is not smooth across $\gamma$. In view of Proposition 1 this is due to the fact that in general the non-zero Lagrange multiplier $\hat{\lambda}$ is concentrated on $\gamma$. This gives rise to a loss of accuracy of numerical methods in a neighborhood of $\gamma$. To overcome this inherent difficulty we 


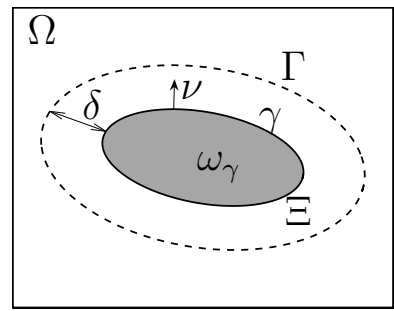

Figure 1: Geometry.

introduce an artificial control boundary $\Gamma \subset \Omega \backslash \bar{\omega}_{\gamma}$ (see Figure 1) and formulate the control problem:

$$
\left.\begin{array}{l}
\text { Find } \mu \in H^{-1 / 2}(\Gamma) \text { such that } u:=u(\mu) \in H_{0}^{1}(\Omega) \text { solves } \\
(\nabla u, \nabla v)_{0, \Omega}=(f, v)_{0, \Omega}+\langle\mu, v\rangle_{\Gamma}, \quad v \in H_{0}^{1}(\Omega), \\
u=g \quad \text { on } \gamma,
\end{array}\right\}
$$

where $\langle,\rangle_{\Gamma}$ denotes the duality pairing between $H^{-1 / 2}(\Gamma)$ and $H^{1 / 2}(\Gamma)$. However this control problem has in general no solution: indeed, since $\mu$ is defined on $\Gamma$ the solution $u(\mu)$ of (3) is smooth in a neighborhood of $\gamma$ by elliptic interior regularity results which is the desired effect. Therefore, $u(\mu)$ does not satisfy the boundary condition $u(\mu)=g \in H^{1 / 2}(\gamma)$ on $\gamma$ in general. But it can be satisfied with an arbitrary accuracy due to the following elliptic approximate controllability result (see $[6])$.

Proposition 2. Define the linear mapping $\phi: H^{-1 / 2}(\Gamma) \rightarrow H^{1 / 2}(\gamma)$ by $\phi(\mu)=$ $\left.u(\mu)\right|_{\gamma}$, where $u(\mu)$ is the solution of $(3)$. Then the range $\phi\left(H^{-1 / 2}(\Gamma)\right)$ is dense in $H^{1 / 2}(\gamma)$.

Remark 1. As a consequence, the range of $\phi$ is also dense in $L^{2}(\gamma)$.

For given $\Gamma \subset \Omega \backslash \bar{\omega}_{\gamma}$ and $\varepsilon>0$ we define the set of admissible controls:

$$
C(\Gamma, \varepsilon)=\left\{\mu \in H^{-1 / 2}(\Gamma):\|u(\mu)-g\|_{0, \gamma} \leq \varepsilon, u(\mu) \text { satisfies }(3)\right\} .
$$

Proposition 3. The set $C(\Gamma, \varepsilon)$ is non-empty, convex and weakly closed for every $\Gamma$ and $\varepsilon>0$.

Proof. Proposition 2 ensures that $C(\Gamma, \varepsilon)$ is non-empty. The rest of the assertion is straightforward. 
Proposition 3 motivates the following auxiliary minimization problem:

$$
\min _{\mu \in C(\Gamma, \varepsilon)} \frac{1}{2}\|\mu\|_{-1 / 2, \Gamma}^{2}
$$

We remark that $(\mathbb{P})_{\Gamma, \varepsilon}$ is a state constrained optimal control problem with $\mu$ as the control variable, the state equation (3) and $\|u(\mu)-g\|_{0, \gamma} \leq \varepsilon$ representing the state constraint. From Proposition 3 we obtain the following existence result.

Proposition 4. Problem $(\mathbb{P})_{\Gamma, \varepsilon}$ has a unique solution for any $\Gamma$ and any $\varepsilon>0$.

Let $\left\{\varepsilon_{n}\right\}$ be an arbitrary sequence of positive numbers tending to zero and $\mu_{n}^{*} \in$ $C\left(\Gamma, \varepsilon_{n}\right)$ be the corresponding solutions of $(\mathbb{P})_{\Gamma, \varepsilon_{n}}$. Then the sequence $\left\{\mu_{n}^{*}\right\}$ is in general unbounded in $H^{-1 / 2}(\Gamma)$. Indeed, if it was bounded one could argue the existence of $\mu^{*} \in C(\Gamma, 0)$, i.e. there would exist a control $\mu^{*}$ such that $u\left(\mu^{*}\right)=g$ holds a.e. on $\gamma$ which in general is not possible.

In order to obtain a sequence of controls which is bounded as $\varepsilon_{n} \rightarrow 0$ we shall in what follows replace the fixed control boundary $\Gamma$ by a sequence $\left\{\Gamma_{n}\right\}$ such that $\Gamma_{n} \rightarrow \gamma$ (in an appropriate way) as $\varepsilon_{n} \rightarrow 0$.

\section{Convergence analysis}

So far $\Gamma$ was fixed. Now we shall allow $\Gamma$ to vary in a set of admissible boundaries specified below. Furthermore, let $G \in H_{0}^{1}(\Omega)$ denote an extension of $g \in H^{1 / 2}(\gamma)$ to $\Omega$. Instead of $(1)$ in $\omega_{\gamma}$ we shall consider a family of problems in $\omega_{\Gamma}$ :

$$
\left.\begin{array}{rll}
-\triangle u_{\Gamma}=f & \text { in } \quad \omega_{\Gamma} \\
u_{\Gamma}=G & \text { on } \quad \Gamma:=\partial \omega_{\Gamma}
\end{array}\right\}
$$

whose fictitious domain formulation reads as follows:

$$
\left.\begin{array}{l}
\text { Find }\left(\hat{u}_{\Gamma}, \hat{\lambda}_{\Gamma}\right) \in H_{0}^{1}(\Omega) \times H^{-1 / 2}(\Gamma) \text { such that } \\
\left(\nabla \hat{u}_{\Gamma}, \nabla v\right)_{0, \Omega}=(f, v)_{0, \Omega}+\left\langle\hat{\lambda}_{\Gamma}, v\right\rangle_{\Gamma} \quad v \in H_{0}^{1}(\Omega), \\
\left\langle\mu, \hat{u}_{\Gamma}\right\rangle_{\Gamma}=\langle\mu, G\rangle_{\Gamma} \quad \mu \in H^{-1 / 2}(\Gamma) .
\end{array}\right\}
$$

The dependence of the solution $\left(\hat{u}_{\Gamma}, \hat{\lambda}_{\Gamma}\right)$ to $(\hat{\mathcal{P}}(\Gamma))$ on $\Gamma$ has been investigated in [5]. Since the techniques of [5] will be used in our subsequent analysis we recall some of the basic results. There we considered domains $\omega_{\Gamma}$ whose boundaries $\Gamma$ are parametrized by curves $\xi:[0,2 \pi] \mapsto \mathbb{R}^{2}$ taken from a set of admissible parametrizations $\mathcal{U}_{a d}$. If $\Gamma$ is parametrized by $\xi$ we shall write $\omega_{\xi}$ instead of $\omega_{\Gamma}$.

The set $\mathcal{U}_{a d}$ was characterized by the following assumptions: 
$(A 1) \xi \in C_{2 \pi}^{2}$ (i.e. $\xi$ is $2 \pi$-periodic and twice differentiable in $[0,2 \pi]$ );

$(A 2)$ there exist positive constants $\alpha, \xi_{1}, \xi_{2}$ independent of $\xi$ such that

$$
\begin{gathered}
|\dot{\xi}(t)| \geq \alpha, \quad t \in[0,2 \pi] \\
|\dot{\xi}|_{\infty} \leq \xi_{1}, \quad|\ddot{\xi}|_{\infty} \leq \xi_{2}
\end{gathered}
$$

(A3) $\xi$ represents a positively oriented curve;

$(A 4) \bar{\omega}_{\xi} \subset \Omega$

$(A 5)$ there exists a positive constant $d>0$ independent of $\xi$ such that

$$
\operatorname{dist}\left(\partial \Omega, \partial \omega_{\xi}\right) \geq d
$$

(A6) there exists a constant $h$ which does not depend on $\xi$ such that for all $t \in[0,2 \pi]$ there are two open discs $B_{i}$ and $B_{o}$ of radius $h$ such that $B_{i} \subset \omega_{\xi}, B_{o} \subset \Omega \backslash \bar{\omega}_{\xi}$ and $\xi(t) \in \bar{B}_{o} \cap \bar{B}_{i}$.

It was shown that $(A 6)$ guarantees the existence of a cut tubular neighborhood $D_{\xi}$ of $\Gamma_{\xi}:=\partial \omega_{\xi}$ given by

$$
D_{\xi}=\{\xi(t)+h \eta \nu(t), \eta \in(-1,1), \quad t \in(0,2 \pi)\}
$$

for some $h>0$ independent of $\xi \in \mathcal{U}_{a d}$. Above $\nu(t)$ denotes the outer normal unit vector to $\Gamma_{\xi}$ at the point $\xi(t)$. For $Q=(0,2 \pi) \times(-1,1)$ the mapping $S_{\xi}^{h}: \bar{Q} \rightarrow \bar{D}_{\xi}$

$$
S_{\xi}^{h}(t, \eta)=\xi(t)+h \eta \nu(t), \quad(t, \eta) \in \bar{Q}
$$

defines a diffeomorphism from $Q$ onto $D_{\xi}$. Replacing $v \in L^{2}\left(\Gamma_{\xi}\right)$ by $v \circ S_{\xi}^{h}(\cdot, 0)$ one obtains a $2 \pi$-periodic function in $L^{2}(0,2 \pi)$. This made it possible to compare functions defined on different boundaries $\Gamma_{\xi}, \xi \in \mathcal{U}_{a d}$. More specifically, we defined the space $L_{2 \pi}^{2}$ as the closure of the space of continuous, $2 \pi$-periodic functions and introduced the subspace

$$
H_{2 \pi}^{1 / 2}=\left\{\varphi \in L_{2 \pi}^{2} \mid\|\varphi\|_{1 / 2,2 \pi}<\infty\right\}
$$

where

$$
\|\varphi\|_{1 / 2,2 \pi}:=\left(|\varphi|_{L_{2 \pi}^{2}}^{2}+\int_{0}^{2 \pi} \int_{0}^{2 \pi} \frac{|\varphi(s)-\varphi(t)|^{2}}{|\sin ((t-s) / 2)|^{2}} d t d s\right)^{1 / 2} .
$$


Using the method of mappings we constructed the trace operators $\mathcal{T}_{\xi}: H_{0}^{1}(\Omega) \rightarrow$ $H_{2 \pi}^{1 / 2}$ which transforms traces on $\Gamma_{\xi}$ into periodic functions from $H_{2 \pi}^{1 / 2}$. It was shown that the family $\left\{\mathcal{T}_{\xi}\right\}_{\xi \in \mathcal{U}_{\text {ad }}}$ is uniformly bounded. Using $\mathcal{T}_{\xi}$ one can relate the spaces $H^{-1 / 2}\left(\Gamma_{\xi}\right)$ and $H_{2 \pi}^{-1 / 2}$ (the dual of $\left.H_{2 \pi}^{1 / 2}\right)$. Indeed, with any $\mu \in H^{-1 / 2}\left(\Gamma_{\xi}\right)$ we associate $\tilde{\mu} \in H_{2 \pi}^{-1 / 2}$ by means of

$$
\left\langle\tilde{\mu}, \mathcal{T}_{\xi} v\right\rangle_{2 \pi}:=\langle\mu, v\rangle_{\Gamma_{\xi}} \quad v \in H_{0}^{1}(\Omega) .
$$

Thus problem $(\hat{\mathcal{P}}(\Gamma))$ can be reformulated as follows:

$$
\left.\begin{array}{l}
\text { Find }\left(\hat{u}_{\xi}, \tilde{\lambda}_{\xi}\right) \in H_{0}^{1}(\Omega) \times H_{2 \pi}^{-1 / 2} \text { such that } \\
\left(\nabla \hat{u}_{\xi}, \nabla v\right)_{0, \Omega}=(f, v)_{0, \Omega}+\left\langle\tilde{\lambda}_{\xi}, \mathcal{T}_{\xi} v\right\rangle_{2 \pi} \quad v \in H_{0}^{1}(\Omega), \\
\left\langle\tilde{\mu}, \mathcal{T}_{\xi} \hat{u}_{\xi}\right\rangle_{2 \pi}=\left\langle\tilde{\mu}, \mathcal{T}_{\xi} G\right\rangle_{2 \pi} \quad \tilde{\mu} \in H_{2 \pi}^{-1 / 2},
\end{array}\right\}
$$

where $\xi \in \mathcal{U}_{a d}$. In [5] we proved the following result (the proof can be readily adapted to cover inhomogeneous boundary conditions too).

Theorem 1. Let $\xi_{n} \rightarrow \xi$ in $C^{1}\left([0,2 \pi], \mathbb{R}^{2}\right), \xi_{n}, \xi \in \mathcal{U}_{\text {ad }}$ and let $\left(\hat{u}_{n}, \tilde{\lambda}_{n}\right) \in H_{0}^{1}(\Omega) \times$ $H_{2 \pi}^{-1 / 2}$ be the solution of $\left(\tilde{\mathcal{P}}\left(\xi_{n}\right)\right)$. Then

$$
\begin{aligned}
& \hat{u}_{n} \rightarrow \hat{u}_{\xi} \text { in } H_{0}^{1}(\Omega) \\
& \tilde{\lambda}_{n} \rightarrow \tilde{\lambda}_{\xi} \text { (weakly) in } H_{2 \pi}^{-1 / 2}, n \rightarrow \infty
\end{aligned}
$$

and $\left(\hat{u}_{\xi}, \tilde{\lambda}_{\xi}\right)$ is the unique solution of $(\tilde{\mathcal{P}}(\xi))$. In addition, if $\xi_{n} \rightarrow \xi$ in $C^{2}\left([0,2 \pi], \mathbb{R}^{2}\right)$ then $\left\{\tilde{\lambda}_{n}\right\}$ converges strongly to $\tilde{\lambda}_{\xi}$ in $H_{2 \pi}^{-1 / 2}$.

Now we return to problem $(\mathbb{P})_{\Gamma, \varepsilon}$. Subsequently we shall assume that the boundary $\gamma$ of $\omega_{\gamma}$ is parameterized by a (fixed) function $\xi \in \mathcal{U}_{a d} \cap C^{3}\left([0,2 \pi] ; \mathbb{R}^{2}\right)$. The control boundary $\Gamma$ is constructed by a shift of $\gamma$ along the normal direction. Thus $\Gamma$ will be parameterized by

$$
\tilde{\xi}(t)=\xi(t)+\delta \nu(t), \quad t \in[0,2 \pi],
$$

for some $\delta \in(0, h)$, where $h$ is specified in $(\mathcal{A} 6)$. Note that

$$
\tilde{\xi}=S_{\xi}^{\delta}(\cdot, 1),
$$

where $S_{\xi}^{\delta}$ is the diffeomorphism defined by (5) with $h:=\delta$. Defining

$$
\mathcal{U}_{a d, \xi}=\{\tilde{\xi}=\xi+\delta \nu, 0<\delta<h\}
$$

and taking $\delta$ sufficiently small one can verify $(\mathcal{A} 1)$ - $(\mathcal{A} 6)$ but with different constants $0<\tilde{\alpha}, \tilde{\xi}_{1}, \tilde{\xi}_{2}$, and $\tilde{d}$ depending on $\xi$ in $(\mathcal{A} 2)$ and $(\mathcal{A} 5)$. Hence Theorem 1 remains valid if $\mathcal{U}_{a d}$ is replaced by $\mathcal{U}_{a d, \xi}$. 
Remark 2. The additional regularity of $\xi$ can be avoided regularizing the normal field $\nu$ in the definition of the diffeomorphism $S_{\xi}^{\delta}$ and in (7). For further details we refer to [1].

Proposition 5. Let $v \in H_{0}^{1}(\Omega)$ and $\Gamma \subset \Omega \backslash \bar{\omega}_{\gamma}$ be parametrized by $\tilde{\xi}(t)=\xi(t)+\delta \nu(t)$, $t \in[0,2 \pi]$, for some $\xi \in \mathcal{U}_{a d}$ and $\delta$ sufficiently small. Then

$$
\|v \circ \tilde{\xi}-v \circ \xi\|_{0,2 \pi} \leq \sqrt{\frac{2}{\alpha}}\|\nabla v\|_{0, \Omega} \sqrt{\delta}
$$

In addition, if $\left.v\right|_{\Gamma}=0$ one has

$$
\|v\|_{0, \gamma} \leq \sqrt{\frac{2 \xi_{1}}{\alpha}}\|\nabla v\|_{0, \Omega} \sqrt{\delta}
$$

with the constants $\alpha$ and $\xi_{1}$ from $(A 2)$.

Proof. It suffices to consider $v \in C^{1}(\bar{\Omega})$. We obtain:

$$
v(\tilde{\xi}(t))-v(\xi(t))=\delta \int_{0}^{1} \nabla v(\xi(t)+\delta \eta \nu(t)) \cdot \nu(t) d \eta=\delta \int_{0}^{1}(\nabla v)\left(S_{\xi}^{\delta}(t, \eta)\right) \cdot \nu(t) d \eta
$$

From $(A 2)$ it follows that

$$
\left|\operatorname{det} D S_{\xi}^{\delta}(t, \eta)\right| \geq \delta(\|\dot{\xi}\|-\delta\|\dot{\nu}\|) \geq \delta \frac{\alpha}{2}
$$

holds for $h$ sufficiently small. Therefore

$$
\begin{aligned}
\| v \circ \tilde{\xi} & -v \circ \xi \|_{0,2 \pi}^{2} \leq \delta^{2} \int_{0}^{2 \pi} \int_{0}^{1}\left|(\nabla v) \circ S_{\xi}^{\delta}\right|^{2} d \eta d t \\
& \leq \delta \frac{2}{\alpha} \int_{0}^{2 \pi} \int_{0}^{1}\left|(\nabla v) \circ S_{\xi}^{\delta}\right|^{2}\left|\operatorname{det} D S_{\xi}^{\delta}(t, \eta)\right| d \eta d t=\delta \frac{2}{\alpha} \int_{D_{\xi}}|\nabla v|^{2} d x \leq \delta \frac{2}{\alpha}\|\nabla v\|_{0, \Omega}^{2} .
\end{aligned}
$$

If $\left.v\right|_{\Gamma}=0$, equivalently if $v \circ \tilde{\xi}=0$, the result follows from

$$
\|v \circ \xi\|_{0,2 \pi} \geq \frac{1}{\sqrt{\xi_{1}}}\|v\|_{0, \gamma}
$$

making use of $(A 2)$ again. 
Using the spaces $H_{2 \pi}^{1 / 2}$ and $H_{2 \pi}^{-1 / 2}$ the state equation in (3) is written as follows:

Given $\tilde{\mu} \in H_{2 \pi}^{-1 / 2}$, find $u_{\tilde{\xi}} \in H_{0}^{1}(\Omega)$ such that

$\left(\nabla u_{\tilde{\xi}}, \nabla v\right)_{0, \Omega}=(f, v)_{0, \Omega}+\left\langle\tilde{\mu}, \mathcal{T}_{\tilde{\xi}} v\right\rangle_{2 \pi}, \quad v \in H_{0}^{1}(\Omega)$,

with $\tilde{\xi}$ defined by $(7)$.

Then the minimization problem $(\mathbb{P})_{\Gamma, \varepsilon}$ can be reformulated as

$$
\left.\min _{\tilde{\mu} \in \tilde{C}(\tilde{\xi}, \varepsilon)} \frac{1}{2}\|\tilde{\mu}\|_{-1 / 2,2 \pi}^{2},\right\}
$$

where

$$
\tilde{C}(\tilde{\xi}, \varepsilon)=\left\{\tilde{\mu} \in H_{2 \pi}^{-1 / 2}:\left\|u_{\tilde{\xi}}-G\right\|_{0, \gamma} \leq \varepsilon, u_{\tilde{\xi}} \text { satisfies }(10)\right\}
$$

Next we shall consider sequences $\varepsilon_{n} \rightarrow 0^{+}$and $\delta_{n} \rightarrow 0^{+}$and establish an a priori bound for the corresponding optimal pairs $\left(u_{n}^{*}, \tilde{\lambda}_{n}^{*}\right)$ of $\left(\tilde{\mathbb{P}}_{\tilde{\xi}_{n}, \varepsilon_{n}}\right.$, where $\tilde{\xi}_{n}$ refers to the parametrization (7) with $\delta$ replaced by $\delta_{n}$.

Proposition 6. Let $\left\{\varepsilon_{n}\right\}$ and $\left\{\delta_{n}\right\}$ converge to zero and let the boundaries $\Gamma_{n}$ and $\gamma$ be parameterized by $\tilde{\xi}_{n}$ and $\xi \in \mathcal{U}_{a d} \cap C^{3}\left([0,2 \pi] ; \mathbb{R}^{2}\right)$, respectively. If $\delta_{n}$ satisfies

$$
\sqrt{\delta_{n}} \hat{c} \leq \varepsilon_{n}, \quad n \in \mathbb{N}
$$

(with a constant $\hat{c}$ specified in (13)), the sequence of optimal pairs $\left\{\left(u_{n}^{*}, \tilde{\lambda}_{n}^{*}\right)\right\}$ of $(\tilde{\mathbb{P}})_{\tilde{\xi}_{n}, \varepsilon_{n}}, n \rightarrow \infty$ is bounded in $H_{0}^{1}(\Omega) \times H_{2 \pi}^{-1 / 2}$.

Proof. Let $\left\{\left(\hat{u}_{n}, \tilde{\lambda}_{n}\right)\right\}$ denote the sequence of the solutions to the fictitious domain problems $\left(\tilde{\mathcal{P}}\left(\tilde{\xi}_{n}\right)\right), n \in \mathbb{N}$. Since $\tilde{\xi}_{n}$ converges to $\xi$ in $C^{1}\left([0,2 \pi], \mathbb{R}^{2}\right)$, Theorem 1 implies the existence of a constant $c_{\lambda}>0$ such that

$$
\left\|\tilde{\lambda}_{n}\right\|_{-1 / 2,2 \pi} \leq c_{\lambda}, \quad n \in \mathbb{N}
$$

Since $\hat{u}_{n}-G=0$ on $\Gamma_{n}$, we infer from (9)

$$
\left\|\hat{u}_{n}-g\right\|_{0, \gamma} \leq \sqrt{\frac{2 \xi_{1}}{\alpha}}\left\|\nabla\left(\hat{u}_{n}-G\right)\right\|_{0, \Omega} \sqrt{\delta_{n}} .
$$

Combined with the estimate

$$
\left\|\nabla \hat{u}_{n}\right\|_{0, \Omega} \leq c_{1}\left(\|f\|_{0, \Omega}+\|\nabla G\|_{0, \Omega}\right), \quad n \in \mathbb{N}
$$


we find that

$$
\left\|\hat{u}_{n}-g\right\|_{0, \gamma} \leq \hat{c} \sqrt{\delta_{n}}
$$

where

$$
\hat{c}=\sqrt{\frac{2 \xi_{1}}{\alpha}}\left(c_{1}\|f\|_{0, \Omega}+\left(1+c_{1}\right)\|\nabla G\|_{0, \Omega}\right) .
$$

Then (11) entails

$$
\left\|\hat{u}_{n}-g\right\|_{0, \gamma} \leq \varepsilon_{n},
$$

hence $\tilde{\lambda}_{n} \in \tilde{C}\left(\tilde{\xi}_{n}, \varepsilon_{n}\right)$. From the definition of $\left(\tilde{\mathbb{P}}_{\tilde{\xi}_{n}, \varepsilon_{n}}\right)$ we have

$$
\left\|\tilde{\lambda}_{n}^{*}\right\|_{-1 / 2,2 \pi} \leq\left\|\tilde{\lambda}_{n}\right\|_{-1 / 2,2 \pi} \leq c_{\lambda} \quad n \in \mathbb{N} .
$$

Let $u_{n}^{*}$ be the solution of the state equation corresponding to $\tilde{\lambda}_{n}^{*}$ :

$$
\left(\nabla u_{n}^{*}, \nabla v\right)_{0, \Omega}=(f, v)_{0, \Omega}+\left\langle\tilde{\lambda}_{n}^{*}, \mathcal{T}_{\tilde{\xi}_{n}} v\right\rangle_{2 \pi} \quad v \in H_{0}^{1}(\Omega) .
$$

Inserting $v:=u_{n}^{*}$, using (14) and the uniform boundedness of $\left(\mathcal{T}_{\tilde{\xi}_{n}}\right)$ (see [5], Lemma 4) one finds that the sequence $\left\{u_{n}^{*}\right\}$ is bounded in $H_{0}^{1}(\Omega)$ as well.

Now we can prove the following convergence result.

Theorem 2. Let all the assumptions of Proposition 6 be satisfied. Then the optimal pairs $\left(u_{n}^{*}, \tilde{\lambda}_{n}^{*}\right)$ of $(\tilde{\mathbb{P}})_{\tilde{\xi}_{n}, \varepsilon_{n}}$ converge strongly in $H_{0}^{1}(\Omega) \times H_{2 \pi}^{-1 / 2}$ to the unique solution $\left(\hat{u}_{\xi}, \tilde{\lambda}_{\xi}\right)$ of the fictitious domain problem $(\tilde{\mathcal{P}}(\xi))$.

Proof. By Proposition 6 there is a subsequence (denoted again by $\left\{\left(u_{n}^{*}, \tilde{\lambda}_{n}^{*}\right)\right\}$ ), which converges weakly in $H_{0}^{1}(\Omega) \times H_{2 \pi}^{-1 / 2}$ to some $\left(\hat{u}_{\xi}, \tilde{\lambda}_{\xi}\right)$. Since $\left(u_{n}^{*}, \tilde{\lambda}_{n}^{*}\right) \in H_{0}^{1}(\Omega) \times H_{2 \pi}^{-1 / 2}$ satisfies

$$
\left(\nabla u_{n}^{*}, \nabla v\right)_{0, \Omega}=(f, v)_{0, \Omega}+\left\langle\tilde{\lambda}_{n}^{*}, \mathcal{T}_{\tilde{\xi}_{n}} v\right\rangle_{2 \pi} \quad v \in H_{0}^{1}(\Omega)
$$

and $\mathcal{T}_{\tilde{\xi}_{n}}$ tends strongly to $\mathcal{T}_{\xi}$ (see [5], Lemma 9) passing to the limit in (15) we deduce

$$
\left(\nabla \hat{u}_{\xi}, \nabla v\right)_{0, \Omega}=(f, v)_{0, \Omega}+\left\langle\tilde{\lambda}_{\xi}, \mathcal{T}_{\xi} v\right\rangle_{2 \pi} \quad v \in H_{0}^{1}(\Omega) .
$$

In view of $\left\|u_{n}^{*}-g\right\|_{0, \gamma} \leq \varepsilon_{n}$ and strong convergence of $u_{n}^{*}$ to $\hat{u}_{\xi}$ in $L^{2}(\gamma)$ we furthermore obtain

$$
\left\|\hat{u}_{\xi}-g\right\|_{0, \gamma}=\lim _{n \rightarrow \infty}\left\|u_{n}^{*}-g\right\|_{0, \gamma}=0 .
$$

This shows that the pair $\left(\hat{u}_{\xi}, \tilde{\lambda}_{\xi}\right)$ solves $(\tilde{\mathcal{P}}(\xi))$. Since this solution is unique, the original sequence $\left\{\left(u_{n}^{*}, \tilde{\lambda}_{n}^{*}\right)\right\}$ converges weakly to $\left(\hat{u}_{\xi}, \tilde{\lambda}_{\xi}\right)$. Taking into account that $\tilde{\xi}_{n} \rightarrow \xi$ in $C^{2}\left([0,2 \pi], \mathbb{R}^{2}\right)$, Theorem 1 implies

$$
\begin{aligned}
\left\|\tilde{\lambda}_{\xi}\right\|_{-1 / 2,2 \pi} & \leq \lim _{n \rightarrow \infty} \inf \left\|\tilde{\lambda}_{n}^{*}\right\|_{-1 / 2,2 \pi} \leq \lim _{n \rightarrow \infty} \sup \left\|\tilde{\lambda}_{n}^{*}\right\|_{-1 / 2,2 \pi} \\
& \leq \lim _{n \rightarrow \infty}\left\|\tilde{\lambda}_{n}\right\|_{-1 / 2,2 \pi}=\left\|\tilde{\lambda}_{\xi}\right\|_{-1 / 2,2 \pi} .
\end{aligned}
$$


Therefore $\lim _{n \rightarrow \infty}\left\|\tilde{\lambda}_{n}^{*}\right\|_{-1 / 2,2 \pi}=\left\|\tilde{\lambda}_{\xi}\right\|_{-1 / 2,2 \pi}$ which ensures strong convergence of $\tilde{\lambda}_{n}^{*}$ to $\tilde{\lambda}_{\xi}$ in $H_{2 \pi}^{-1 / 2}$.

We are now able to prove strong convergence of $\hat{u}_{n}^{*}$ to $\hat{u}_{\xi}$ in $H_{0}^{1}(\Omega)$. In fact, the difference $\hat{u}_{n}^{*}-\hat{u}_{n}$ satisfies (recall that $\left(\hat{u}_{n}, \tilde{\lambda}_{n}\right)$ is the solution of the fictitious domain problem $\left.\left(\mathcal{P}\left(\tilde{\xi}_{n}\right)\right)\right)$

$$
\left(\nabla\left(\hat{u}_{n}^{*}-\hat{u}_{n}\right), \nabla v\right)_{0, \Omega}=\left\langle\tilde{\lambda}_{n}^{*}-\tilde{\lambda}_{n}, \mathcal{T}_{\tilde{\xi}_{n}} v\right\rangle_{2 \pi}, \quad v \in H_{0}^{1}(\Omega)
$$

so that

$$
\left\|\hat{u}_{n}^{*}-\hat{u}_{n}\right\|_{H_{0}^{1}(\Omega)} \leq c\left\|\tilde{\lambda}_{n}^{*}-\tilde{\lambda}_{n}\right\|_{-1 / 2,2 \pi}\left\|\mathcal{T}_{\tilde{\xi}_{n}}\right\|
$$

with a constant $c>0$ which does not depend on $n$. This shows $\hat{u}_{n}^{*}-\hat{u}_{n} \rightarrow 0$ strongly in $H_{0}^{1}(\Omega)$ using the boundedness of the sequence $\left\{\mathcal{T}_{\tilde{\xi}_{n}}\right\}$. Now, the convergence result follows from the triangle inequality

$$
\left\|\hat{u}_{n}^{*}-\hat{u}_{\xi}\right\|_{H_{0}^{1}(\Omega)} \leq\left\|\hat{u}_{n}^{*}-\hat{u}_{n}\right\|_{H_{0}^{1}(\Omega)}+\left\|\hat{u}_{n}-\hat{u}_{\xi}\right\|_{H_{0}^{1}(\Omega)}
$$

and Theorem 1.

\section{A penalty approach}

We consider at first a fixed control boundary $\Gamma$. The state constraint $\|u-g\|_{0, \gamma} \leq \varepsilon$ in problem $(\mathbb{P})_{\Gamma, \varepsilon}$ is numerically realized by a penalty approach. We introduce for $k>0$ the augmented cost functional

$$
J_{k}(u, \lambda)=\frac{1}{2}\|\lambda\|_{-1 / 2, \Gamma}^{2}+\frac{k}{2}\|u-g\|_{0, \gamma}^{2},
$$

and define the new problem

$$
\left.\begin{array}{l}
\min J_{k}(u, \lambda) \text { subject to } \\
\quad(u, \lambda) \in H_{0}^{1}(\Omega) \times H^{-1 / 2}(\Gamma), \\
\quad(\nabla u, \nabla v)_{0, \Omega}=(f, v)_{0, \Omega}+\langle\lambda, v\rangle_{\Gamma}, \quad v \in H_{0}^{1}(\Omega),
\end{array}\right\}
$$

which has a unique solution denoted by $\left(w_{k}, \sigma_{k}\right)$. In view of (12) one obtains by an argument adapted from [9] the estimate

$$
J_{k}\left(w_{k}, \sigma_{k}\right) \leq J_{k}\left(\hat{u}_{\Gamma}, \hat{\lambda}_{\Gamma}\right) \leq \frac{1}{2}\left\|\hat{\lambda}_{\Gamma}\right\|_{-1 / 2, \Gamma}^{2}+\frac{k}{2} \hat{c}^{2} \delta
$$

where $\left(\hat{u}_{\Gamma}, \hat{\lambda}_{\Gamma}\right)$ is the solution of $(\hat{\mathcal{P}}(\Gamma))$. This implies

$$
\frac{k}{2}\left\|w_{k}-g\right\|_{0, \gamma}^{2} \leq \frac{1}{2}\left\|\hat{\lambda}_{\Gamma}\right\|_{-1 / 2, \Gamma}^{2}+\frac{k}{2} \hat{c}^{2} \delta
$$


so that

$$
\left\|w_{k}-g\right\|_{0, \gamma}^{2} \leq \frac{\left\|\hat{\lambda}_{\Gamma}\right\|_{-1 / 2, \Gamma}^{2}}{k}+\hat{c}^{2} \delta .
$$

Choosing

$$
k \geq \frac{\left\|\hat{\lambda}_{\Gamma}\right\|_{-1 / 2, \Gamma}^{2}}{\hat{c}^{2} \delta}
$$

we obtain

$$
\left\|w_{k}-g\right\|_{0, \gamma} \leq \sqrt{2} \hat{c} \sqrt{\delta}
$$

In order to ensure at least weak convergence of the optimal pairs $\left(w_{k}, \sigma_{k}\right), k \rightarrow \infty$ we again introduce a sequence of the control boundaries $\Gamma_{n}$ parametrized by $(7)$ with $\delta$ replaced by $\delta_{n}$. We select for each $\Gamma_{n}$ a penalty parameter $k_{n}>0$ such that

$$
\frac{\left\|\hat{\lambda}_{n}\right\|_{-1 / 2, \Gamma_{n}}^{2}}{\hat{c}^{2}} \leq \delta_{n} k_{n} \leq \kappa,
$$

where $\left(\hat{u}_{n}, \hat{\lambda}_{n}\right) \in H_{0}^{1}(\Omega) \times H^{1 / 2}\left(\Gamma_{n}\right)$ is the solution of the fictitious domain formulation $\left(\hat{\mathcal{P}}\left(\Gamma_{n}\right)\right)$ and $\kappa>0$ is fixed. Such a choice of $\kappa$ is possible due to the boundedness of $\left\{\hat{\lambda}_{n}\right\}$. Then the sequence $\left\{J_{k_{n}}\left(w_{k_{n}}, \sigma_{k_{n}}\right)\right\}$ is bounded by

$$
J_{k_{n}}\left(w_{k_{n}}, \sigma_{k_{n}}\right) \leq \frac{1}{2}\left\|\hat{\lambda}_{n}\right\|_{-1 / 2, \Gamma_{n}}^{2}+\frac{1}{2} \kappa \hat{c}^{2} .
$$

which yields the boundedness of $\sigma_{k_{n}}$ in $H^{-1 / 2}\left(\Gamma_{n}\right), n \in \mathbb{N}$. Let $\tilde{\sigma}_{k_{n}} \in H_{2 \pi}^{-1 / 2}$ stand for the functional $\sigma_{k_{n}}$ interpreted on $H_{2 \pi}^{1 / 2}$. As in the proof of Theorem 2 one concludes the existence of a subsequence of $\left\{\left(w_{k_{n}}, \tilde{\sigma}_{k_{n}}\right)\right\}$ which converges weakly in $H_{0}^{1}(\Omega) \times H_{2 \pi}^{-1 / 2}$ to a pair $(w, \tilde{\sigma})$ such that

$$
(\nabla w, \nabla v)_{0, \Omega}=(f, v)_{0, \Omega}+\left\langle\tilde{\sigma}, \mathcal{T}_{\xi} v\right\rangle_{2 \pi}, \quad v \in H_{0}^{1}(\Omega) .
$$

In view of (16) we also have

$$
\left\|w_{k_{n}}-g\right\|_{0, \gamma} \leq \sqrt{2} \hat{c} \sqrt{\delta_{n}}
$$

so that $w$ satisfies the original boundary condition $w=g$ on $\gamma$. Hence $(w, \tilde{\sigma})$ is a solution of the fictitious domain problem $(\tilde{\mathcal{P}}(\xi))$. Consequently, $(w, \tilde{\sigma})=\left(\hat{u}_{\xi}, \tilde{\lambda}_{\xi}\right)$ must hold. In addition, the (whole) sequence $\left\{\left(\hat{w}_{k_{n}}, \tilde{\sigma}_{k_{n}}\right)\right\}$ tends weakly in $H_{0}^{1}(\Omega) \times$ $H_{2 \pi}^{-1 / 2}$ to $\left(\hat{u}_{\xi}, \tilde{\lambda}_{\xi}\right)$.

We now turn to the first order necessary and sufficient optimality condition for problem $(\mathbb{P})_{\Gamma, k}$ for a fixed control boundary $\Gamma$. Let $S \in \mathcal{L}\left(H^{-1 / 2}(\Gamma), L^{2}(\gamma)\right)$ denote 
the Poincare-Steklov like operator $S \lambda=\tau_{\gamma} u$ where $u \in H_{0}^{1}(\Omega)$ is the unique solution of

$$
(\nabla u, \nabla v)_{0, \Omega}=\left\langle\lambda, \tau_{\Gamma} v\right\rangle_{\Gamma}, \quad v \in H_{0}^{1}(\Omega),
$$

where $\tau_{\gamma}, \tau_{\Gamma}$ are the trace mappings from $H_{0}^{1}(\Omega)$ into $L^{2}(\gamma)$ and $H^{1 / 2}(\Gamma)$, respectively. If $A \in \mathcal{L}\left(H_{0}^{1}(\Omega), H^{-1}(\Omega)\right)$ denotes the isomorphism induced by the inner product in $H_{0}^{1}(\Omega)$, one can represent $S$ as

$$
S=\tau_{\gamma} A^{-1} \tau_{\Gamma}^{*}
$$

Thus one can formulate the equivalent problem in terms of $\lambda$ :

$$
\min _{\lambda \in H^{-1 / 2}(\Gamma)} \tilde{J}_{k}(\lambda)=\frac{1}{2}\|\lambda\|_{-1 / 2, \Gamma}^{2}+\frac{k}{2}\|S \lambda-d\|_{0, \gamma}^{2}
$$

with

$$
d=g-\tau_{\gamma} A^{-1} f
$$

We endow $H^{1 / 2}(\Gamma)$ with the norm

$$
\|\varphi\|_{1 / 2, \Gamma}=\inf \left\{\|\nabla v\|_{0, \Omega}: \tau_{\Gamma} v=\varphi, v \in H_{0}^{1}(\Omega)\right\}, \quad \varphi \in H^{1 / 2}(\Gamma)
$$

which is induced by the inner product

$$
(\varphi, \psi)_{1 / 2, \Gamma}=\left(\nabla y_{\varphi}, \nabla y_{\psi}\right)_{0, \Omega}, \quad \varphi, \psi \in H^{1 / 2}(\Gamma) .
$$

Here $y_{\varphi}$ (and analogously $\left.y_{\psi}\right)$ is the first component of the solution $\left(y_{\varphi}, \lambda_{\varphi}\right) \in$ $H_{0}^{1}(\Omega) \times H^{-1 / 2}(\Gamma)$ to the saddle-point problem

$$
\begin{aligned}
& \left(\nabla y_{\varphi}, \nabla v\right)_{0, \Omega}-\left\langle\lambda_{\varphi}, \tau_{\Gamma} v\right\rangle_{\Gamma}=0, \quad v \in H_{0}^{1}(\Omega), \\
& \left\langle\mu, \tau_{\Gamma} y_{\varphi}\right\rangle_{\Gamma}=\langle\mu, \varphi\rangle_{\Gamma}, \quad \mu \in H^{-1 / 2}(\Gamma) .
\end{aligned}
$$

Thus the Riesz mapping $R: H^{1 / 2}(\Gamma) \rightarrow H^{-1 / 2}(\Gamma)$ is given by

$$
\langle R \varphi, \psi\rangle_{\Gamma}=\left(\nabla y_{\varphi}, \nabla y_{\psi}\right)_{0, \Omega}
$$

From this one deduces that the Riesz representation $\varphi_{\lambda} \in H^{1 / 2}(\Gamma)$ of $\lambda \in H^{-1 / 2}(\Gamma)$ is $\varphi_{\lambda}=\tau_{\Gamma} y_{R^{-1} \lambda}$, where $y_{R^{-1} \lambda}$ is the unique solution of

$$
\left(\nabla y_{R^{-1} \lambda}, \nabla v\right)_{0, \Omega}=\left\langle\lambda, \tau_{\Gamma} v\right\rangle_{\Gamma}, \quad v \in H_{0}^{1}(\Omega)
$$

which implies

$$
R^{-1} \lambda=\tau_{\Gamma} A^{-1} \tau_{\Gamma}^{*} \lambda
$$


Consequently, the norm in $H^{-1 / 2}(\Gamma)$ can be realized by

$$
\|\lambda\|_{-1 / 2, \Gamma}=\left\|\nabla y_{R^{-1} \lambda}\right\|_{0, \Omega}
$$

and the inner product in $H^{-1 / 2}(\Gamma)$ by

$$
(\lambda, \varrho)_{-1 / 2, \Gamma}=\left(R^{-1} \lambda, R^{-1} \varrho\right)_{1 / 2, \Gamma}=\left(\nabla y_{R^{-1} \lambda}, \nabla y_{R^{-1}}\right)_{0, \Omega}, \quad \lambda, \varrho \in H^{-1 / 2}(\Gamma) .
$$

Thus the derivative of the reduced cost functional $J_{k}$ is given by

$$
\left\langle\tilde{J}_{k}^{\prime}(\lambda), \varrho\right\rangle_{H^{1 / 2}(\Gamma), H^{-1 / 2}(\Gamma)}=(\lambda, \varrho)_{-1 / 2, \Gamma}+k(S \lambda-d, S \varrho)_{0, \gamma}
$$

which leads to the optimality condition

$$
\left(R^{-1}+k S^{*} S\right) \lambda=k S^{*} d
$$

in $H^{1 / 2}(\Gamma)$. We note that one evaluation of $\left(R^{-1}+k S^{*} S\right) \lambda$ requires the solution of two elliptic equations. Indeed, solving the system

$$
\begin{aligned}
& A y=\tau_{\Gamma}^{*} \lambda, \\
& A z=\tau_{\gamma}^{*} \tau_{\gamma} y
\end{aligned}
$$

one finds that

$$
\left(R^{-1}+k S^{*} S\right) \lambda=\tau_{\Gamma} y+k \tau_{\Gamma} z .
$$

We remark that to solve the equations in (21) is numerically inexpensive.

\section{Numerical examples}

In the following, we address two aspects of the smooth embedding method based on the penalty approach introduced in the previous section. Firstly, we illustrate a higher accuracy of this approach compared to the classical fictitious domain method [3]. Secondly, we demonstrate its computational efficiency when solving large scale problems (up to 16 millions unknowns). We shall consider the model example

$$
\left.\begin{array}{rl}
-\triangle u=f & \text { in } \quad \omega \\
u=g & \text { on } \quad \gamma=\partial \omega .
\end{array}\right\}
$$

The domain $\omega$ is represented by the interior of the ellipse

$$
\omega=\left\{(x, y) \in \mathbb{R}^{2} \mid \frac{(x-0.5)^{2}}{0.4^{2}}+\frac{(y-0.5)^{2}}{0.2^{2}}<1\right\}
$$




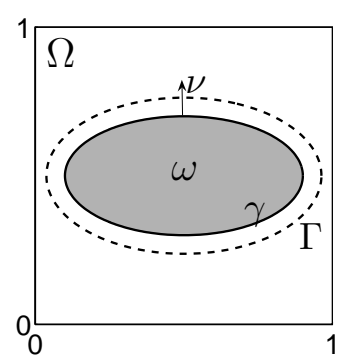

Figure 2: Geometry of $\omega$ and $\Omega$.

embedded in the unit square $\Omega=(0,1) \times(0,1)$ (see Figure 2). The right hand-sides $f$ and $g$ in $(\mathcal{P}(\omega))$ are $f=-\Delta \hat{u}$ in $\mathbb{R}^{2}$ and $g=\left.\hat{u}\right|_{\gamma}$, where

$$
\hat{u}(x, y)=e^{y} \sin (\pi(x-y)),(x, y) \in \mathbb{R}^{2} .
$$

Thus exact solution $u_{e x}$ of $(\mathcal{P}(\omega))$ is given by $\hat{u}_{\left.\right|_{\omega}}$.

To discretize the space $H_{0}^{1}(\Omega)$ we use the subspace $V_{h}$ of all continuous piecewise bilinear functions vanishing on $\partial \Omega$ constructed over a uniform partition of $\Omega$ into squares of size $h$. The spaces $H^{-1 / 2}(\gamma)$ and $H^{-1 / 2}(\Gamma)$ are approximated by the subspaces $\Lambda_{H_{\gamma}}$ and $\Lambda_{H_{\Gamma}}$ of functions which are piecewise constant on partitions $\mathcal{T}_{H_{\gamma}}$, $\mathcal{T}_{H_{\Gamma}}$ of polygonal approximations of $\gamma$ and $\Gamma$, respectively. We emphasize that the meshnorms $H_{\gamma}, H_{\Gamma}$ of $\mathcal{T}_{H_{\gamma}}$ and $\mathcal{T}_{H_{\Gamma}}$, respectively, may be chosen independently in contrast to the approach in [6].

The resulting algebraic formulation of (20) is given by

$$
\left(\mathbf{R}^{-1}+k \mathbf{S}^{T} \mathbf{S}\right) \boldsymbol{\lambda}=k \mathbf{S}^{T} \mathbf{d},
$$

where

$$
\mathbf{R}^{-1}=\mathbf{B}_{\Gamma} \mathbf{A}^{-1} \mathbf{B}_{\Gamma}^{T}, \quad \mathbf{S}=\mathbf{B}_{\gamma} \mathbf{A}^{-1} \mathbf{B}_{\Gamma}^{T}
$$

and $\mathbf{A} \in \mathbb{R}^{n \times n}$ is the stiffness matrix, $\mathbf{B}_{\Gamma} \in \mathbb{R}^{m_{\Gamma} \times n}$ and $\mathbf{B}_{\gamma} \in \mathbb{R}^{m_{\gamma} \times n}$ are the matrices representing the discrete trace mappings on $\Gamma$ and $\gamma$, respectively, $n=\operatorname{dim} V_{h}$, $m_{\Gamma}=\operatorname{dim} \Lambda_{H_{\Gamma}}$, and $m_{\gamma}=\operatorname{dim} \Lambda_{H_{\gamma}}$. Finally, $\mathbf{d}=\mathbf{g}-\mathbf{B}_{\gamma} \mathbf{A}^{-1} \mathbf{f} \in \mathbb{R}^{m_{\gamma}}$, where $\mathbf{f} \in \mathbb{R}^{n}$ denotes the discrete load vector and $\mathbf{g} \in \mathbb{R}^{m_{\gamma}}$ is a piecewise constant approximation of $g$. More details about the discretization may be found in [6].

The algebraic system (22) is solved by the conjugate gradient method with the relative stopping tolerance $\epsilon=h^{2}$ and zero as the initial guess. Analogously to the continuous setting one multiplication of the system matrix with the vector $\boldsymbol{\lambda}$ in (22) 
requires the solution of the following two systems:

$$
\begin{aligned}
\mathbf{A y} & =\mathbf{B}_{\Gamma}^{T} \boldsymbol{\lambda}, \\
\mathbf{A z} & =\mathbf{B}_{\gamma}^{T} \mathbf{B}_{\gamma} \mathbf{y},
\end{aligned}
$$

so that

$$
\left(\mathbf{R}^{-1}+k \mathbf{S}^{T} \mathbf{S}\right) \boldsymbol{\lambda}=\mathbf{B}_{\Gamma} \mathbf{y}+k \mathbf{B}_{\Gamma} \mathbf{z} .
$$

Finally, to solve the system (23) at a low cost we replace the space $H_{0}^{1}(\Omega)$ by $H_{p e r}^{1}(\Omega)$, i.e. the subspace of functions in $H^{1}(\Omega)$ which are periodic. The theoretical results of the previous sections remain valid also in this case. Then $V_{h} \subset H_{p e r}^{1}(\Omega)$ will be the space of all continuous piecewise bilinear functions having the same traces on opposite sides of $\Omega$. The advantage of this choice consists in the fact that the resulting stiffness matrix has a circulant structure which enables the use of the highly efficient Poisson like solver ([8], [6]) based on the discrete Fourier transform.

In the first numerical experiment we compare the classical fictitious domain approach $(\hat{\mathcal{P}}(\Gamma))$ with our smooth embedding domain method. We approximate at first the solution $u_{e x}$ of $(\mathcal{P}(\omega))$ by the finite element approximations of $\left.\hat{u}_{\Gamma}\right|_{\omega}$ where $\hat{u}_{\Gamma}$ is the first component of the solution to $(\hat{\mathcal{P}}(\Gamma))$ for different control boundaries $\Gamma$. The Dirichlet boundary condition for $\hat{u}_{\Gamma}$ on $\Gamma$ is determined by $\hat{u}_{\Gamma} \circ \tilde{\xi}=g \circ \xi$ where $\xi$ and $\tilde{\xi}$ are the parametrizations of $\gamma$ and $\Gamma$, respectively. In Table 1 we report the resulting relative errors of the finite element solution $\hat{u}_{h}$ corresponding to the fixed meshsize $h=1 / 256$ in the following norms

$\operatorname{Err}_{L^{2}(\omega)}=\frac{\left\|\hat{u}_{h}-u_{e x}\right\|_{L^{2}(\omega)}}{\left\|u_{e x}\right\|_{L^{2}(\omega)}}, \operatorname{Err}_{H^{1}(\omega)}=\frac{\left\|\hat{u}_{h}-u_{e x}\right\|_{H^{1}(\omega)}}{\left\|u_{e x}\right\|_{H^{1}(\omega)}}, \operatorname{Err}_{L^{2}(\gamma)}=\frac{\left\|\hat{u}_{h}-u_{e x}\right\|_{L^{2}(\gamma)}}{\left\|u_{e x}\right\|_{L^{2}(\gamma)}}$

decreasing the distance $\delta$ between the boundary $\Gamma$ and the original boundary $\gamma$ from $\delta=8 h$ to $\delta=0$. We also record $n=\operatorname{dim} V_{h}, m_{\Gamma}=\operatorname{dim} \Lambda_{H_{\Gamma}}, m_{\gamma}=\operatorname{dim} \Lambda_{H_{\gamma}}$, the number of $\mathrm{CG}$ iterations and the computing time.

From Theorem 1 we know that if $\Gamma$ is close to $\gamma$ (in the appropriate sense) then also the solution $\left(\hat{u}_{\Gamma}, \hat{\lambda}_{\Gamma}\right)$ of $(\hat{\mathcal{P}}(\Gamma))$ is close to the solution $\left(\hat{u}_{\gamma}, \hat{\lambda}_{\gamma}\right)$ of $(\hat{\mathcal{P}}(\gamma))$. It is not surprising that the best result is obtained for $\delta=0$, i.e. $\gamma \equiv \Gamma$, as seen from Table 1 . This can be explained observing that $\left.\hat{u}\right|_{\omega}$ solves the original problem $(\mathcal{P}(\omega))$ and that in particular $\hat{u}=g$ is satisfied exactly on $\gamma$ whereas the function $\hat{u}_{\Gamma}$ satisfies the same boundary condition on $\Gamma$ but not on $\gamma$. The longer the distance of $\Gamma$ from $\gamma$ is the worse solution one obtains. On the other hand if the smooth variant of the fictitious domain method is used the behaviour is opposite: results are better for increasing distances of $\Gamma$ from $\gamma$. Table 2 illustrates that the Dirichlet condition on $\gamma$ is satisfied with a higher accuracy than in the previous case while 
the singularity of the solution to the state problem which is concentrated on $\Gamma$ is sufficiently far away from $\gamma$.

It has been already mentioned that the approach in [6] is based on the assumption that $\operatorname{dim} \Lambda_{H_{\Gamma}}=\operatorname{dim} \Lambda_{H_{\gamma}}$. This restriction is not needed in the new approach any more. Hence, the number of controls on $\Gamma$ may be smaller than the number of arcs used to discretize $\gamma$. This can improve the conditioning of the system matrix when $m_{\Gamma}<m_{\gamma}$. In Tables 3 and 4 we report similar data as before for two different choices of the penalty parameter $k$. The distance of the control boundary to the original boundary is $\delta=8 h$, we set $m_{\gamma}=2 m_{\Gamma}$ and decrease the meshsize $h$ from $1 / 128$ to $1 / 4096$. For $k=100 / h^{2}$ one observes the optimal convergence rate in $L^{2}(\omega)$ and $H^{1}(\omega)$. However, the number of CG iterations and accordingly the computing time increase with $n=\operatorname{dim} V_{h}$. This drawback can be significantly reduced by the nested iterations technique which uses a hierarchy of nested grids. The solution of the smooth embedding domain method on a coarse level is used as an initial guess for the CG method on the next finer level (see [6] for more details). The results for this case are reported in Table 5.

Table 1: Classical FD approach applied to problems defined in $\omega_{\Gamma}$

\begin{tabular}{|c|c|c|c|c|c|c|}
\hline$\delta$ & $n /\left(m_{\Gamma}=m_{\gamma}\right)$ & Iters. & S.time $[\mathrm{s}]$ & $\operatorname{Err}_{L^{2}(\omega)}$ & $\operatorname{Err}_{H^{1}(\omega)}$ & $\operatorname{Err}_{L^{2}(\gamma)}$ \\
\hline $8 h$ & $66049 / 62$ & 12 & 0.4836 & $1.2463 \mathrm{e}-001$ & $1.9095 \mathrm{e}-001$ & $1.3455 \mathrm{e}-001$ \\
$4 h$ & $66049 / 62$ & 13 & 0.4836 & $6.7877 \mathrm{e}-002$ & $1.7496 \mathrm{e}-001$ & $7.8189 \mathrm{e}-002$ \\
$2 h$ & $66049 / 62$ & 13 & 0.5616 & $3.7895 \mathrm{e}-002$ & $1.9069 \mathrm{e}-001$ & $5.6803 \mathrm{e}-002$ \\
$1 h$ & $66049 / 62$ & 12 & 0.4992 & $1.9722 \mathrm{e}-002$ & $1.5933 \mathrm{e}-001$ & $3.7727 \mathrm{e}-002$ \\
$0 h$ & $66049 / 62$ & 11 & 0.4368 & $2.4426 \mathrm{e}-003$ & $4.9886 \mathrm{e}-002$ & $2.2412 \mathrm{e}-003$ \\
\hline
\end{tabular}

Table 2: Smooth penalty approach $\left(k=10^{2} / h^{2}\right)$

\begin{tabular}{|c|c|c|c|c|c|c|}
\hline$\delta$ & $n /\left(m_{\Gamma}=m_{\gamma}\right)$ & Iters. & S.time $[\mathrm{s}]$ & $\operatorname{Err}_{L^{2}(\omega)}$ & $\operatorname{Err}_{H^{1}(\omega)}$ & $\operatorname{Err}_{L^{2}(\gamma)}$ \\
\hline $8 h$ & $66049 / 62$ & 25 & 0.9516 & $1.3087 \mathrm{e}-005$ & $1.1775 \mathrm{e}-003$ & $1.8968 \mathrm{e}-005$ \\
$4 h$ & $66049 / 62$ & 14 & 0.5148 & $2.0114 \mathrm{e}-005$ & $1.5453 \mathrm{e}-003$ & $7.8423 \mathrm{e}-005$ \\
$2 h$ & $66049 / 62$ & 13 & 0.4992 & $4.8910 \mathrm{e}-005$ & $2.9809 \mathrm{e}-003$ & $2.3736 \mathrm{e}-004$ \\
$1 h$ & $66049 / 62$ & 11 & 0.4368 & $1.4074 \mathrm{e}-004$ & $7.5131 \mathrm{e}-003$ & $6.4170 \mathrm{e}-004$ \\
$0 h$ & $66049 / 62$ & 11 & 0.4524 & $2.4426 \mathrm{e}-003$ & $4.9886 \mathrm{e}-002$ & $2.2412 \mathrm{e}-003$ \\
\hline
\end{tabular}


Table 3: Smooth penalty approach with $k=1 / h^{2}$

\begin{tabular}{|c|c|c|c|c|c|c|}
\hline Step $h$ & $n / m_{\Gamma} / m_{\gamma}$ & Iters. & S.time $[\mathrm{s}]$ & $\operatorname{Err}_{L^{2}(\omega)}$ & $\operatorname{Err}_{H^{1}(\omega)}$ & $\operatorname{Err}_{L^{2}(\gamma)}$ \\
\hline $1 / 128$ & $16641 / 18 / 36$ & 12 & 0.15 & $1.7625 \mathrm{e}-02$ & $6.7895 \mathrm{e}-02$ & $2.8024 \mathrm{e}-02$ \\
$1 / 256$ & $66049 / 31 / 62$ & 19 & 0.67 & $4.4524 \mathrm{e}-03$ & $2.4064 \mathrm{e}-02$ & $6.3279 \mathrm{e}-03$ \\
$1 / 512$ & $263169 / 55 / 110$ & 28 & 4.84 & $1.5542 \mathrm{e}-03$ & $1.3141 \mathrm{e}-02$ & $2.0537 \mathrm{e}-03$ \\
$1 / 1024$ & $1050625 / 99 / 198$ & 52 & 34.17 & $6.2905 \mathrm{e}-04$ & $8.2158 \mathrm{e}-03$ & $8.0627 \mathrm{e}-04$ \\
$1 / 2048$ & $4198401 / 180 / 360$ & 82 & 261.3 & $2.7170 \mathrm{e}-04$ & $5.3690 \mathrm{e}-03$ & $3.4317 \mathrm{e}-04$ \\
$1 / 4096$ & $16785409 / 331 / 662$ & 170 & 2634.7 & $1.2181 \mathrm{e}-04$ & $3.5870 \mathrm{e}-03$ & $1.5273 \mathrm{e}-04$ \\
\hline \multicolumn{7}{|c|}{ Convergence rates: } \\
\hline
\end{tabular}

Table 4: Smooth penalty approach with $k=10^{2} / h^{2}$

\begin{tabular}{|c|c|c|c|c|c|c|}
\hline Step $h$ & $n / m_{\Gamma} / m_{\gamma}$ & Iters. & S.time $[\mathrm{s}]$ & $\operatorname{Err}_{L^{2}(\omega)}$ & $\operatorname{Err}_{H^{1}(\omega)}$ & $\operatorname{Err}_{L^{2}(\gamma)}$ \\
\hline $1 / 128$ & $16641 / 18 / 36$ & 12 & 0.15 & $1.9692 \mathrm{e}-03$ & $2.3229 \mathrm{e}-02$ & $5.3687 \mathrm{e}-03$ \\
\hline $1 / 256$ & $66049 / 31 / 62$ & 19 & 0.73 & $1.4821 \mathrm{e}-04$ & $4.6439 \mathrm{e}-03$ & $5.1751 \mathrm{e}-04$ \\
\hline $1 / 512$ & $263169 / 55 / 110$ & 30 & 5.33 & $3.0126 \mathrm{e}-05$ & $1.9723 \mathrm{e}-03$ & $1.2875 \mathrm{e}-04$ \\
\hline $1 / 1024$ & $1050625 / 99 / 198$ & 50 & 32.67 & $9.5058 \mathrm{e}-06$ & $1.0753 \mathrm{e}-03$ & $4.5825 \mathrm{e}-05$ \\
\hline $1 / 2048$ & $4198401 / 180 / 360$ & 85 & 282.0 & $3.1593 \mathrm{e}-06$ & $6.0307 \mathrm{e}-04$ & $1.4711 \mathrm{e}-05$ \\
\hline $1 / 4096$ & $16785409 / 331 / 662$ & 170 & 2622.0 & $1.2982 \mathrm{e}-06$ & $3.8004 \mathrm{e}-04$ & $5.7447 \mathrm{e}-06$ \\
\hline \multicolumn{4}{|c|}{ Convergence rates: } & 2.033 & 1.125 & 1.893 \\
\hline
\end{tabular}

Table 5: Smooth penalty approach with $k=10^{2} / h^{2}$ with nested iterations

\begin{tabular}{|c|c|c|c|c|c|c|}
\hline Step $h$ & $n / m_{\Gamma} / m_{\gamma}$ & Iters. & S.time $[\mathrm{s}]$ & $\operatorname{Err}_{L^{2}(\omega)}$ & $\operatorname{Err}_{H^{1}(\omega)}$ & $\operatorname{Err}_{L^{2}(\gamma)}$ \\
\hline $1 / 128$ & $16641 / 18 / 36$ & 12 & 0.22 & $1.9629 \mathrm{e}-03$ & $2.3141 \mathrm{e}-02$ & $5.3546 \mathrm{e}-03$ \\
$1 / 256$ & $66049 / 32 / 64$ & 22 & 0.94 & $1.3408 \mathrm{e}-04$ & $4.3896 \mathrm{e}-03$ & $4.7949 \mathrm{e}-04$ \\
$1 / 512$ & $263169 / 56 / 112$ & 27 & 5.10 & $2.7062 \mathrm{e}-05$ & $1.8580 \mathrm{e}-03$ & $1.1596 \mathrm{e}-04$ \\
$1 / 1024$ & $1050625 / 100 / 200$ & 32 & 30.67 & $8.1246 \mathrm{e}-06$ & $9.8913 \mathrm{e}-04$ & $3.8310 \mathrm{e}-05$ \\
$1 / 2048$ & $4198401 / 180 / 360$ & 36 & 146.4 & $3.0539 \mathrm{e}-06$ & $5.9225 \mathrm{e}-04$ & $1.3877 \mathrm{e}-05$ \\
$1 / 4096$ & $16785409 / 330 / 660$ & 45 & 958.7 & $1.2377 \mathrm{e}-06$ & $3.6990 \mathrm{e}-04$ & $4.9212 \mathrm{e}-06$ \\
\hline \multicolumn{7}{|c|}{ Convergence rates: } \\
\hline
\end{tabular}




\section{Conclusions}

To improve the convergence rate of the standard fictitious domain method with boundary Lagrange multipliers we proposed a smooth variant of this approach given by state constrained optimal control problems. The control variables are represented by forces concentrated on a closed curve $\Gamma$ surrounding the original domain $\omega$. By means of them we control the behavior of the solution $u$ to the state problem. Our goal was to find a control with the smallest norm such that the error between $u$ and the prescribed Dirichlet data on $\gamma$ (measured in the $L^{2}(\gamma)$-norm) is below a prescribed tolerance $\varepsilon$. To treat this state constraint numerically we used the penalty approach. The respective necessary and sufficient optimality condition leads to a linear equation in the space of controls. Its discretization by finite elements was used for finding the (unique) discrete optimal solution. The improvement of the convergence rate in comparison to the standard fictitious domain method is illustrated with a model example.

Acknowledgement This research was supported by the grant IAA1075402 of the Grant Agency of the Czech Academy of Sciences and by the Research Project MSM0021620839 of the Czech Ministry of Education.

\section{References}

[1] A.Boulkhemair, A. Chakib and A. Nachaoui (2008). Continuity of the trace operator with respect to the domain and application to shape optimization. Appl.Comp.Math. 7:192-205.

[2] F. Brezzi and M.Fortin (1991). Mixed and Hybrid Finite Element Methods. Springer Series in Computational Mathematics, Vol.15, Springer- Verlag, New York.

[3] V. Girault and R. Glowinski (1995). Error analysis of a fictitious domain method applied to a Dirichlet problem. Japan J. Indust. Appl. Math. 12:487514 .

[4] J.Haslinger, T.Kozubek, K.Kunisch and G.Peichl (2003). Shape Optimization and Fictitious Domain Approach for Solving Free Boundary Problems of Bernoulli type. Comput.Optimiz.and Appl. 26:231-251.

[5] J. Haslinger, T. Kozubek, K. Kunisch and G. Peichl (2004). An embedding domain approach for a class of 2-d shape optimization problems: mathematical analysis. J. Math. Anal. Appl. 290:665-685. 
[6] J. Haslinger, T. Kozubek, R. Kučera, and G. Peichl (2007). Projected Schur complement method for solving non-symmetric systems arising from a smooth fictitious domain approach. Numerical Linear Algebra with Applications 14:713-739.

[7] J.Haslinger and R. Makinen (2003). Introduction to Shape Optimization, Theory, Approximation and Computation. SIAM, Advances in Design and Control.

[8] R. Kučera (2005). Complexity of an algorithm for solving saddle-point systems with singular blocks arising in wavelet-Galerkin discretizations. Appl. Math. 50:291-308.

[9] R. Glowinski, J.L. Lions and J. He (2008). Exact and approximate controllability for distributed parameter systems: a numerical approach. Cambridge University Press, Cambridge. 